Article

\title{
Similarity Measures for T-Spherical Fuzzy Sets with Applications in Pattern Recognition
}

\author{
Kifayat Ullah* (D), Tahir Mahmood and Naeem Jan \\ Department of Mathematics \& Statistics, International Islamic University, Islamabad 44000, Pakistan; \\ tahirbakhat@iiu.edu.pk (T.M.); naeem.phdma73@iiu.edu.pk (N.J.) \\ * Correspondence: kifayat.phdma72@iiu.edu.pk
}

Received: 13 May 2018; Accepted: 24 May 2018; Published: 1 June 2018

\begin{abstract}
In this manuscript, two generalizations of fuzzy sets, intuitionistic fuzzy sets and picture fuzzy sets, known as spherical fuzzy sets and T-spherical fuzzy sets, are discussed and a numerical and geometrical comparison among them is established. A T-spherical fuzzy set can model phenomena like voting using four characteristic functions denoting the degree of vote in favor, abstinence, vote in opposition, and refusal with an infinite domain, whereas an intuitionistic fuzzy set can model only phenomena of yes or no types. First, in this manuscript, some similarity measures in the frameworks of intuitionistic fuzzy sets and picture fuzzy sets are discussed. With the help of some numerical results, it is discussed that existing similarity measures have some limitations and could not be applied to problems where information is provided in T-spherical fuzzy environment. Therefore, some new similarity measures in the framework of spherical fuzzy sets and T-spherical fuzzy sets are proposed including cosine similarity measures, grey similarity measures, and set theoretic similarity measures. With the help of some results, it was proved that the proposed similarity measures are a generalization of existing similarity measures. The newly-defined similarity measures were subjected to a well-known problem of building material recognition and the results are discussed. A comparative study of new and existing similarity measures was established and some advantages of the proposed work are discussed.
\end{abstract}

Keywords: intuitionistic fuzzy set; picture fuzzy set; spherical fuzzy set; T-spherical fuzzy set; similarity measures; pattern recognition

\section{Introduction}

The study of fuzzy set (FS) theory was initiated in Reference [1] where the membership degree $s$ of an element of a set was defined by a characteristic function on a unit interval $[0,1]$ and the non-membership degree could be obtained by subtracting membership grade form 1 . Atanassov [2] extended Zadeh's concept of FS to intuitionistic fuzzy set (IFS) where the membership and non-membership degrees, i.e., $s$ and $d$, are defined independently but with a constraint that their sum must belong to the interval $[0,1]$, i.e., $\operatorname{sum}(s, d) \in[0,1]$. Further, the term $1-\operatorname{sum}(s, d)$ was referred to as hesitancy degree. Due to the restriction of Atanassov's model of IFS, values cannot be assigned to its characteristic functions, as sometimes the $\operatorname{sum}(s, d)$ exceeds $[0,1]$ interval. Therefore, Yager [3,4] proposed the framework of Pythagorean FSs, enlarging the domain of IFSs as the constraint of Pythagorean FS becomes sum $\left(s^{2}, d^{2}\right) \in[0,1]$. The domain of characteristic functions of IFS and Pythagorean FSs are depicted in Figure 1. For some developments in these areas we refer readers to References [5-10]. 


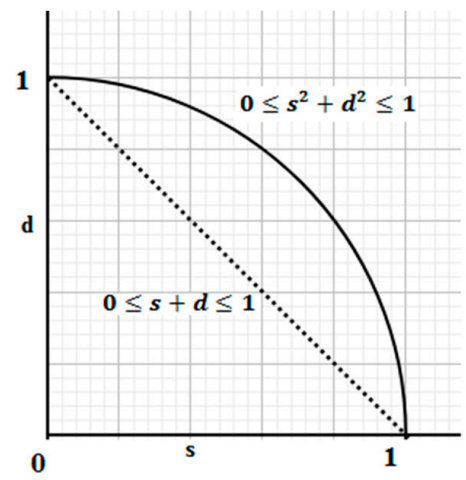

Figure 1. Comparison of spaces of intuitionistic fuzzy sets and Pythagorean fuzzy sets [9].

Atanassov's model of IFSs successfully improved Zadeh's model of FSs, but in a scenario when there are more than two independent situations like in voting (vote in favor, abstinence, vote against and refusal), IFSs failed to describe the situation. Realizing this, in Reference [11], Cuong developed a structure known as a picture fuzzy set (PFS), having three characteristic functions denoted by $s, i$ and $d$ with a condition that their sum must belong to $[0,1]$, i.e., $\operatorname{sum}(s, i, d) \in[0,1]$. The term $1-\operatorname{sum}(s, i, d)$ was referred to as the refusal degree of an element of PFSs. The domain of characteristic functions of PFSs is depicted in Figure 2. For some relevant work one may refer to [12-17].

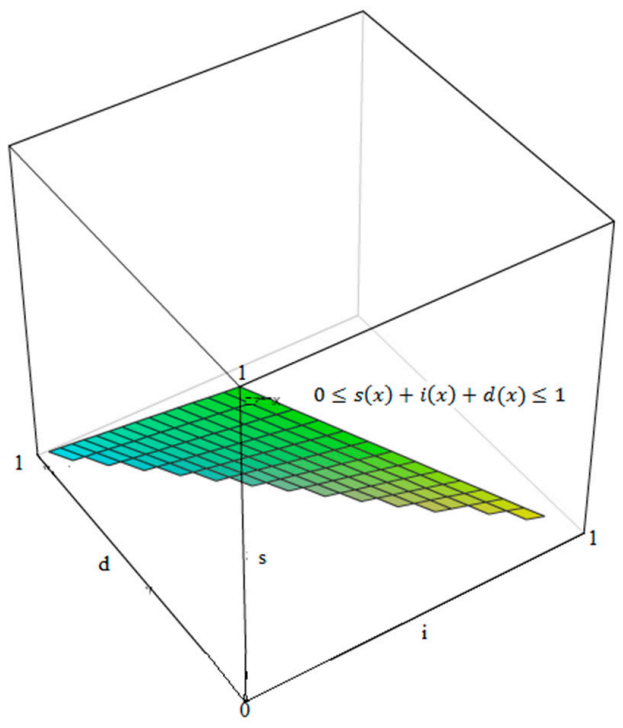

Figure 2. Space of pictorial membership grades [18].

PFSs extended the frameworks of FSs and IFSs, but still there is a limitation of this structure, i.e., the domain of PFSs is restricted and grades of characteristic functions cannot be assigned independently. Observing the structure of [2,3,11], Mahmood et al. [18] proposed a novel framework of spherical fuzzy set (SFS) as a generalization of PFSs by increasing the domain of PFSs. In the structure of SFSs the characteristic functions are denoted by $s, i$ and $d$ with a constraint that the sum of $s, i$ and $d$ may exceeded from the unit interval but their square must belong to the unit interval, i.e., sum $\left(s^{2}, i^{2}, d^{2}\right) \in[0,1]$. This new constraint made the domain of SFSs larger than PFSs as depicted in Figure 3. Sometimes, if $s=0.7, i=0.6$ and $d=0.9$ is assumed, even squaring is not enough because $\operatorname{sum}(0.7,0.6,0.9)=1.66 \not \leq 1$. To deal with such situations, Mahmood et al. [18] proposed a generalization of SFSs known as T-spherical fuzzy set (TSFS) which has no limitation at all as a TSFS is characterized by $s, i$ and $d$ with a condition that $\operatorname{sum}\left(s^{n}, i^{n}, d^{n}\right) \in[0,1]$ where $n \in \mathbb{Z}$. Regarding this new constraint, for a triplet $(0.7,0.6,0.9)$ and $n=5$ : 
$\operatorname{sum}\left(0.7^{5}, 0.6^{5}, 0.9^{5}\right)=0.83632 \in[0,1]$. This example supported the claim of Mahmood et al. indicating that TSFS is a generalization of IFS, PFS and SFS having no limitations. A comparison of PFSs with SFSs and TSFSs is shown in Figures 3-5.

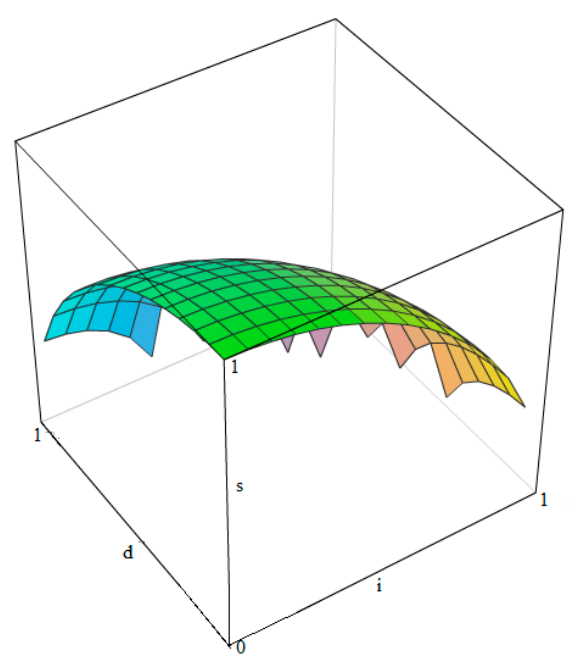

Figure 3. Space of spherical membership grades [18].

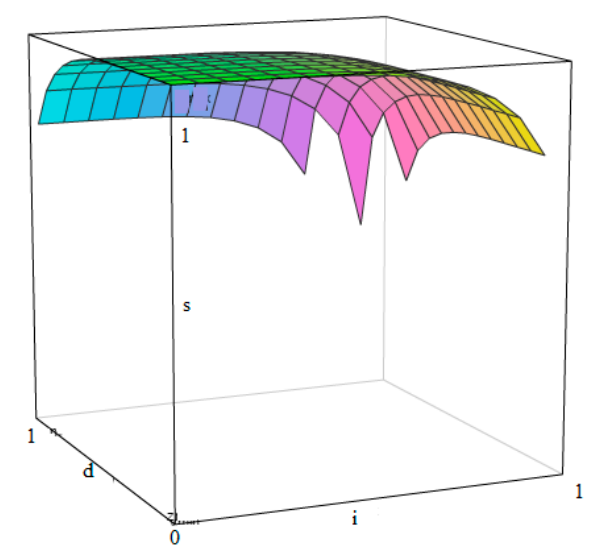

Figure 4. Space of T-spherical membership for $n=5$ [18].

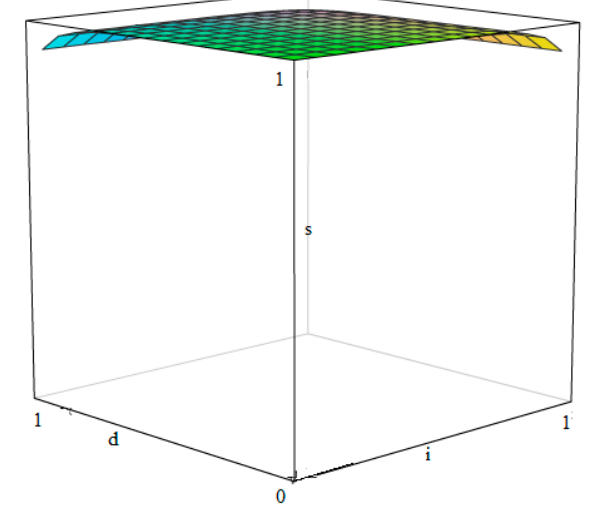

Figure 5. Space of T-spherical membership for $n=10$ [18].

The aim of this article was to develop some similarity measures for the defined TSFSs. A similarity measure provided the similarity degree of two objects i.e., how similar two objects are. The study of 
similarity measures in the framework of FSs [1] and IFSs [2] has an extensive history as it is widely applied in cluster analysis [19,20], data mining [21,22], pattern recognition [23-29], information retrieval [30-32], medical diagnosis [33], and multi-attribute decision making [34] etc. Due to its application in different situations, considerable attention has been given to similarity measures. $\mathrm{Xu}$ and Chen [35] provided an overview of similarity measures of IFSs, Xu discussed correlation coefficients for IFS [36], the authors of [37] studied similarity measures of hesitant fuzzy sets (HFSs), Reference [14] investigated correlation coefficients for PFSs, Reference [38] focused on applications of correlation coefficients, and Reference [14,39-41] discussed several similarity and distance measures and studied some correlation coefficients for PFSs.

In this manuscript, it is observed that the similarity measures developed in References $[28,37,40]$ have some limitations and cannot be applied to problems where information is provided in T-spherical fuzzy environment. To resolve this issue, some novel similarity measuring tools including cosine similarity measures, set-theoretic similarity measures, and grey similarity measures are proposed for TSFSs which are generalizations of the similarity measures developed in References [28,37,40]. It is also proposed that existing similarity measures become special cases of the developed similarity measures showing the novelty and diversity of proposed similarity measures. A building material recognition problem was solved by applying the new similarity measures and the results are discussed.

This article is divided into different sections. In Section 1, some prerequisites of the proposed work have been comparatively discussed. In Section 2, some very basic definitions are studied. In Section 3, new similarity measures are defined including cosine similarity measures, set-theoretic similarity measures and grey similarity measures. Section 4 is based on the application of proposed similarity measures in pattern recognition where a building material recognition problem is solved. The article ends with a comparative analysis of existing and new work along with some limitations of existing work and advantages of the work proposed are discussed.

\section{Preliminaries}

In this section, some notions of IFSs, Pythagorean FSs, PFSs, SFSs and TSFSs are comparatively examined. It is discussed how TSFSs generalize all existing structures. The notions discussed in this section provided a foundation for our work. For undefined terms and notions, one may refer to References $[1-4,28,37,40]$.

Definition 1. [2] An IFS on a universe of discourse $X$ is of the form $I=\{\langle x, s(x), d(x)\rangle\}$ such that s and $d$ denote the affiliation and non-affiliation of $x \in X$ respectively on a unit interval $[0,1]$ and $0 \leq \operatorname{Sum}(s, d) \leq 1$ and $r(x)=1-\operatorname{Sum}(s, d)$ is the hesitancy degree of $x$ in $S$. The duplet $(s, d)$ is considered as an intuitionistic fuzzy number (IFN).

Definition 2. [3] A Pythagorean FS on a universe of discourse $X$ is of the form $P=\{\langle x, s(x), d(x)\rangle\}$ such that $s$ and $d$ denote the affiliation and non-affiliation of $x \in X$ respectively on a unit interval $[0,1]$ and $0 \leq \operatorname{Sum}\left(s^{2}, d^{2}\right) \leq 1$ and $r(x)=1-\operatorname{Sum}\left(s^{2}, d^{2}\right)$ is the hesitancy degree of $x$ in $S$. The duplet $(s, d)$ is considered as a Pythagorean fuzzy number.

Definition 3. [11] A PFS on a universe of discourse $X$ is of the form $P=\{\langle x, s(x), i(x), d(x)\rangle\}$ such that $s, i$ and $d$ denote the affiliation, abstinence and non-affiliation of $x \in X$ respectively on a unit interval $[0,1]$ and $0 \leq \operatorname{Sum}(s, i, d) \leq 1$ and $r(x)=1-\operatorname{Sum}(s, i, d)$ is the refusal degree of $x$ in $S$. The triplet $(s, i, d)$ is considered as a picture fuzzy number (PFN).

Definition 4. [18] A SFS on a universe of discourse $X$ is of the form $S=\{\langle x, s(x), i(x), d(x)\rangle\}$ such that $s, i$ and $d$ denote the affiliation, abstinence and non-affiliation of $x \in X$ respectively on a unit interval $[0,1]$ and $0 \leq \operatorname{Sum}\left(s^{2}, i^{2}, d^{2}\right) \leq 1$ and $r(x)=\sqrt{1-\operatorname{Sum}\left(s^{2}, i^{2}, d^{2}\right)}$ is the refusal degree of $x$ in $S$. The triplet $(s, i, d)$ is considered as a spherical fuzzy number (SFN). 
Definition 5. [18] A TSFS on a universe of discourse $X$ is of the form $P=\{\langle x, s(x), i(x), d(x)\rangle\}$ such that $s, i$ and $d$ denote the affiliation, abstinence and non-affiliation of $x \in X$ respectively on a unit interval $[0,1]$ and $0 \leq \operatorname{Sum}\left(s^{n}, i^{n}, d^{n}\right) \leq 1$ for $n \in \mathbb{Z}$ and $r(x)=\sqrt[n]{1-\operatorname{Sum}\left(s^{n}, i^{n}, d^{n}\right)}$ is the refusal degree of $x$ in $S$. The triplet $(s, i, d)$ is considered as a T-spherical fuzzy number (TSFN).

Remark 1. In Definition 5, if

- We place $n=2$ then TSFS becomes SFS.

- We place $n=1$ then TSFS becomes PFS.

- We place $n=2$ and $i=0$ then TSFS becomes Pythagorean FS.

- We place $n=1$ and $i=0$ then TSFS becomes IFS.

- We place $n=1$ and $i=d=0$ then TSFS becomes FS.

This proved that the frame work of TSFS is the most generalized among the existing structures.

Theorem 1. [18] The SFSs have a larger space of than that of space of PFSs.

Theorem 2. [18] The TSFSs have a larger space of than that of space of SFSs.

Theorem 3. [18] The TSFSs have a larger space of than that of space of PFSs.

Definition 6. [28] For two IFNs $P=\left(s_{P}, d_{P}\right)$ and $Q=\left(s_{Q}, d_{Q}\right)$ on a universe of discourse $X$, a cosine similarity measure is defined as:

$$
C_{\text {IFS }}^{1}(P, Q)=\frac{1}{m} \sum_{i=1}^{m} \frac{s_{P}\left(x_{i}\right) \cdot s_{Q}\left(x_{i}\right)+d_{P}\left(x_{i}\right) \cdot d_{Q}\left(x_{i}\right)}{\sqrt{s_{P}^{2}\left(x_{i}\right)+d_{P}^{2}\left(x_{i}\right)} \cdot \sqrt{s_{Q}^{2}\left(x_{i}\right)+d_{Q}^{2}\left(x_{i}\right)}}
$$

Definition 7. [37] For two IFNs $P=\left(s_{P}, d_{P}\right)$ and $Q=\left(s_{Q}, d_{Q}\right)$ on a universe of discourse $X$ and $i=1-s-d$, a set-theoretic similarity measure is defined as:

$$
C_{\text {IFS }}^{2}(P, Q)=\frac{1}{m} \sum_{i=1}^{m} \frac{s_{P}\left(x_{i}\right) \cdot s_{Q}\left(x_{i}\right)+i_{P}\left(x_{i}\right) \cdot i_{Q}\left(x_{i}\right)+d_{P}\left(x_{i}\right) \cdot d_{Q}\left(x_{i}\right)}{\max \left(s_{P}^{2}\left(x_{i}\right)+d_{P}^{2}\left(x_{i}\right)+i_{P}^{2}\left(x_{i}\right), s_{Q}^{2}\left(x_{i}\right)+d_{Q}^{2}\left(x_{i}\right)+i_{Q}^{2}\left(x_{i}\right)\right)}
$$

Definition 8. [37] For two IFNs $P=\left(s_{P}, d_{P}\right)$ and $Q=\left(s_{Q}, d_{Q}\right)$ on a universe of discourse $X$ and $i=1-s-d$, the grey similarity measure is defined as:

$$
C_{I F S}^{3}(P, Q)=\frac{1}{3 m} \sum_{i=1}^{m}\left(\frac{\Delta s_{\min }+\Delta s_{\max }}{\Delta s_{i}+\Delta s_{\max }}+\frac{\Delta d_{\min }+\Delta d_{\max }}{\Delta d_{i}+\Delta d_{\max }}\right)
$$

where $\Delta s_{i}=\left|s_{P}\left(x_{i}\right)-s_{Q}\left(x_{i}\right)\right|$ and $\Delta d_{i}=\left|d_{P}\left(x_{i}\right)-d_{Q}\left(x_{i}\right)\right|$ further $\Delta s_{\min }=\min \left\{\left|s_{P}\left(x_{i}\right)-s_{Q}\left(x_{i}\right)\right|\right\}$ and $\Delta d_{\text {min }}=\min \left\{\left|d_{P}\left(x_{i}\right)-d_{Q}\left(x_{i}\right)\right|\right\}$ also $\Delta s_{\max }=\max \left\{\left|s_{P}\left(x_{i}\right)-s_{Q}\left(x_{i}\right)\right|\right\}$ and $\Delta d_{\max }=$ $\max \left\{\left|d_{P}\left(x_{i}\right)-d_{Q}\left(x_{i}\right)\right|\right\}$.

Definition 9. [40] For two PFNs $P=\left(s_{P}, i_{P}, d_{P}\right)$ and $Q=\left(s_{Q}, i_{Q}, d_{Q}\right)$ on a universe of discourse $X, a$ cosine similarity measure is defined as:

$$
C_{P F S}^{1}(P, Q)=\frac{1}{m} \sum_{i=1}^{m} \frac{s_{P}\left(x_{i}\right) \cdot s_{Q}\left(x_{i}\right)+i_{P}\left(x_{i}\right) \cdot i_{Q}\left(x_{i}\right)+d_{P}\left(x_{i}\right) \cdot d_{Q}\left(x_{i}\right)}{\sqrt{s_{P}^{2}\left(x_{i}\right)+i_{P}^{2}\left(x_{i}\right)+d_{P}^{2}\left(x_{i}\right)} \cdot \sqrt{s_{Q}^{2}\left(x_{i}\right)+i_{Q}^{2}\left(x_{i}\right)+d_{Q}^{2}\left(x_{i}\right)}}
$$


Definition 10. [40] For two PFNs $P=\left(s_{P}, i_{P}, d_{P}\right)$ and $Q=\left(s_{Q}, i_{Q}, d_{Q}\right)$ on a universe of discourse $X, a$ set-theoretic similarity measure is defined as:

$$
C_{P F S}^{2}(P, Q)=\frac{1}{m} \sum_{i=1}^{m} \frac{s_{P}\left(x_{i}\right) \cdot s_{Q}\left(x_{i}\right)+i_{P}\left(x_{i}\right) \cdot i_{Q}\left(x_{i}\right)+d_{P}\left(x_{i}\right) \cdot d_{Q}\left(x_{i}\right)}{\max \left(s_{P}^{2}\left(x_{i}\right)+i_{P}^{2}\left(x_{i}\right)+d_{P}^{2}\left(x_{i}\right), s_{Q}^{2}\left(x_{i}\right)+i_{Q}^{2}\left(x_{i}\right)+d_{Q}^{2}\left(x_{i}\right)\right)}
$$

Definition 11. [40] For two PFNs $P=\left(s_{P}, i_{P}, d_{P}\right)$ and $Q=\left(s_{Q}, i_{Q}, d_{Q}\right)$ on a universe of discourse $X$, the grey similarity measure is defined as:

$$
C_{P F S}^{3}(P, Q)=\frac{1}{3 m} \sum_{i=1}^{m}\left(\frac{\Delta s_{\min }+\Delta s_{\max }}{\Delta s_{i}+\Delta s_{\max }}+\frac{\Delta i_{\min }+\Delta i_{\max }}{\Delta i_{i}+\Delta i_{\max }}+\frac{\Delta d_{\min }+\Delta d_{\max }}{\Delta d_{i}+\Delta d_{\max }}\right)
$$

where $\Delta s_{i}=\left|s_{P}\left(x_{i}\right)-s_{Q}\left(x_{i}\right)\right|, \Delta i_{i}=\left|i_{P}\left(x_{i}\right)-i_{Q}\left(x_{i}\right)\right|$ and $\Delta d_{i}=\left|d_{P}\left(x_{i}\right)-d_{Q}\left(x_{i}\right)\right|$ further $\Delta s_{\text {min }}=$ $\min \left\{\left|s_{P}\left(x_{i}\right)-s_{Q}\left(x_{i}\right)\right|\right\}, \Delta i_{\text {min }}=\min \left\{\left|i_{P}\left(x_{i}\right)-i_{Q}\left(x_{i}\right)\right|\right\}$ and $\Delta d_{\min }=\min \left\{\left|d_{P}\left(x_{i}\right)-d_{Q}\left(x_{i}\right)\right|\right\}$ also $\Delta s_{\max }=\max \left\{\left|s_{P}\left(x_{i}\right)-s_{Q}\left(x_{i}\right)\right|\right\}, \Delta i_{\max }=\max \left\{\left|i_{P}\left(x_{i}\right)-i_{Q}\left(x_{i}\right)\right|\right\}$ and $\Delta d_{\max }=$ $\max \left\{\left|d_{P}\left(x_{i}\right)-d_{Q}\left(x_{i}\right)\right|\right\}$.

The similarity measures discussed in this section are limited and can handle the data provided in the framework of IFSs or PFSs. Therefore, in this manuscript, some new similarity measures in the environment of TSFSs shall be proposed as a generalization of similarity measures defined in Equations (1)-(6).

\section{Similarity Measures}

In this section, some similarity measures are developed in the environment of TSFSs including cosine similarity measure, grey similarity measure, and set theoretic similarity measures adopted from $[28,37,40]$. It is proved that the similarity measures developed here are generalizations of existing similarity measures (Equations (1)-(6)) and can also be applied to those areas where existing similarity measures failed to be applied.

\subsection{Cosine Similarity Measures}

Following the work of $[28,37,40]$, we developed similarity measures for TSFSs. We claimed that the proposed similarity measures are generalizations of the similarity measures discussed in Equations (1) and (4).

Definition 12. For two TSFNs $P=\left(s_{P}, i_{P}, d_{P}\right)$ and $Q=\left(s_{Q}, i_{Q}, d_{Q}\right)$ on a universe of discourse $X, a$ cosine similarity measure is defined as:

$$
C_{T S F S}^{1}(P, Q)=\frac{1}{m} \sum_{i=1}^{m} \frac{s_{P}^{n}\left(x_{i}\right) \cdot s_{Q}^{n}\left(x_{i}\right)+i_{P}^{n}\left(x_{i}\right) \cdot i_{Q}^{n}\left(x_{i}\right)+d_{P}^{n}\left(x_{i}\right) \cdot d_{Q}^{n}\left(x_{i}\right)}{\sqrt{\left(s_{P}^{n}\left(x_{i}\right)\right)^{2}+\left(i_{P}^{n}\left(x_{i}\right)\right)^{2}+\left(d_{P}^{n}\left(x_{i}\right)\right)^{2}} \cdot \sqrt{\left(s_{Q}^{n}\left(x_{i}\right)\right)^{2}+\left(i_{Q}^{n}\left(x_{i}\right)\right)^{2}+\left(d_{Q}^{n}\left(x_{i}\right)\right)^{2}}}
$$

The following properties hold true for the cosine similarity measure of three TSFNs $P=\left(s_{P}, i_{P}, d_{P}\right)$, $Q=\left(s_{Q}, i_{Q}, d_{Q}\right)$ and $R=\left(s_{R}, i_{R}, d_{R}\right)$.

1. $0 \leq C_{T S F S}^{1}(P, Q) \leq 1$.

2. $C_{\text {TSFS }}^{1}(P, Q)=C_{T S F S}^{1}(Q, P)$.

3. $C_{T S F S}^{1}(P, Q)=1$ if $P=Q$ and $i=1,2,3, \ldots m$.

4. If $P \sqsubseteq Q \sqsubseteq R$. Then $C_{\text {TSFS }}^{1}(P, R) \leq C_{\text {TSFS }}^{1}(P, Q), C_{\text {TSFS }}^{1}(P, R) \leq C_{\text {TSFS }}^{1}(Q, R)$. 
Proof. The proofs of first and second are obvious. To prove the third part, take $P=Q$ i.e., $\mathrm{s}_{P}\left(x_{i}\right)=\mathrm{s}_{Q}\left(x_{i}\right), i_{P}\left(x_{i}\right)=i_{Q}\left(x_{i}\right), d_{P}\left(x_{i}\right)=d_{Q}\left(x_{i}\right)$, hence Equation (7) implies

$$
\begin{aligned}
C_{T S F S}^{1}(\mathrm{P}, \mathrm{Q}) & =\frac{1}{m} \sum_{i=1}^{m} \frac{s_{\mathrm{P}}^{\mathrm{n}}\left(x_{i}\right) \cdot s_{P}^{\mathrm{n}}\left(x_{i}\right)+i_{\mathrm{P}}^{\mathrm{n}}\left(x_{i}\right) \cdot i_{P}^{\mathrm{n}}\left(x_{i}\right)+d_{\mathrm{P}}^{\mathrm{n}}\left(x_{i}\right) \cdot d_{P}^{\mathrm{n}}\left(x_{i}\right)}{\sqrt{\left(s_{\mathrm{P}}^{\mathrm{n}}\left(x_{i}\right)\right)^{2}+\left(i_{\mathrm{P}}^{\mathrm{n}}\left(x_{i}\right)\right)^{2}+\left(d_{\mathrm{P}}^{\mathrm{n}}\left(x_{i}\right)\right)^{2}} \cdot \sqrt{\left(s_{P}^{\mathrm{n}}\left(x_{i}\right)\right)^{2}+\left(i_{\mathrm{P}}^{\mathrm{n}}\left(x_{i}\right)\right)^{2}+\left(d_{P}^{\mathrm{n}}\left(x_{i}\right)\right)^{2}}} \\
& =\frac{1}{m} \sum_{i=1}^{m} \frac{\left(s_{\mathrm{P}}^{\mathrm{n}}\left(x_{i}\right)\right)^{2}+\left(i_{\mathrm{P}}^{\mathrm{n}}\left(x_{i}\right)\right)^{2}+\left(d_{\mathrm{P}}^{\mathrm{n}}\left(x_{i}\right)\right)^{2}}{\left(s_{\mathrm{P}}^{\mathrm{n}}\left(x_{i}\right)\right)^{2}+\left(i_{\mathrm{P}}^{\mathrm{n}}\left(x_{i}\right)\right)^{2}+\left(d_{\mathrm{P}}^{\mathrm{n}}\left(x_{i}\right)\right)^{2}} \\
& =1
\end{aligned}
$$

The fourth part is obvious as geometrically, the angle of $P, R$ is greater than that of $P, Q$ and $Q, R$.

Definition 13. The distance measure of the angle between two TSFNs P and $Q$ is defined as:

$$
\mathrm{d}^{\prime}(P, Q)=\arccos \left(C_{\text {TSFS }}^{1}(\mathrm{P}, \mathrm{Q})\right)
$$

The following properties hold true for the distance measure of three TSFNS $P=\left(s_{P}, i_{P}, d_{P}\right)$, $Q=\left(s_{Q}, i_{Q}, d_{Q}\right)$ and $R=\left(s_{R}, i_{R}, d_{R}\right)$.

1. If $0 \leq C_{T S F S}^{1}(P, Q) \leq 1$. Then $\mathrm{d}^{\prime}(P, Q) \geq 0$.

2. If $C_{\text {TSFS }}^{1}(P, Q)=C_{\text {TSFS }}^{1}(Q, P)$. Then $\mathrm{d}^{\prime}(P, Q)=\mathrm{d}^{\prime}(Q, P)$.

3. If $C_{T S F S}^{1}(P, Q)=1$ for $P=Q$ and $i=1,2,3, \ldots m$. Then $\mathrm{d}^{\prime}(P, Q)=0$.

4. If $P \sqsubseteq Q \sqsubseteq R$. Then $\mathrm{d}^{\prime}(P, R) \leq \mathrm{d}^{\prime}(P, Q)+\mathrm{d}^{\prime}(Q, R)$.

Proof. The proofs of Part (1) - (3) are obvious. To prove Part (4), let $P \sqsubseteq Q \sqsubseteq R$. Then the distance measures of $P, Q$ and $R$ are:

$$
\begin{aligned}
& \mathrm{d}^{\prime}\left(P\left(x_{i}\right), Q\left(x_{i}\right)\right)=\arccos \left(C_{\text {TSFS }}^{1}\left(P\left(x_{i}\right), Q\left(x_{i}\right)\right)\right) \\
& \mathrm{d}^{\prime}\left(Q\left(x_{i}\right), R\left(x_{i}\right)\right)=\arccos \left(C_{T S F S}^{1}\left(Q\left(x_{i}\right), R\left(x_{i}\right)\right)\right) \\
& \mathrm{d}^{\prime}\left(P\left(x_{i}\right), R\left(x_{i}\right)\right)=\arccos \left(C_{T S F S}^{1}\left(P\left(x_{i}\right), R\left(x_{i}\right)\right)\right)
\end{aligned}
$$

where $i=1,2,3, \ldots m$ and

$$
\begin{aligned}
& C_{T S F S}^{1}\left(P\left(x_{i}\right), Q\left(x_{i}\right)\right)=\frac{1}{m} \sum_{i=1}^{m} \frac{s_{P}^{n}\left(x_{i}\right) \cdot s_{Q}^{n}\left(x_{i}\right)+i_{P}^{n}\left(x_{i}\right) \cdot i_{Q}^{n}\left(x_{i}\right)+d_{P}^{n}\left(x_{i}\right) \cdot d_{Q}^{n}\left(x_{i}\right)}{\sqrt{\left(s_{P}^{n}\left(x_{i}\right)\right)^{2}+\left(i_{P}^{n}\left(x_{i}\right)\right)^{2}+\left(d_{P}^{n}\left(x_{i}\right)\right)^{2}} \cdot \sqrt{\left(s_{Q}^{n}\left(x_{i}\right)\right)^{2}+\left(i_{Q}^{n}\left(x_{i}\right)\right)^{2}+\left(d_{Q}^{n}\left(x_{i}\right)\right)^{2}}} \\
& C_{\text {TSFS }}^{1}\left(Q\left(x_{i}\right), R\left(x_{i}\right)\right)=\frac{1}{m} \sum_{i=1}^{m} \frac{s_{Q}^{n}\left(x_{i}\right) \cdot s_{R}^{n}\left(x_{i}\right)+i_{Q}^{n}\left(x_{i}\right) \cdot i_{R}^{n}\left(x_{i}\right)+d_{Q}^{n}\left(x_{i}\right) \cdot d_{R}^{n}\left(x_{i}\right)}{\sqrt{\left(s_{Q}^{n}\left(x_{i}\right)\right)^{2}+\left(i_{Q}^{n}\left(x_{i}\right)\right)^{2}+\left(d_{Q}^{n}\left(x_{i}\right)\right)^{2}} \cdot \sqrt{\left(s_{R}^{n}\left(x_{i}\right)\right)^{2}+\left(i_{R}^{n}\left(x_{i}\right)\right)^{2}+\left(d_{R}^{n}\left(x_{i}\right)\right)^{2}}} \\
& C_{T S F S}^{1}\left(P\left(x_{i}\right), R\left(x_{i}\right)\right)=\frac{1}{m} \sum_{i=1}^{m} \frac{s_{P}^{n}\left(x_{i}\right) \cdot s_{R}^{n}\left(x_{i}\right)+i_{P}^{n}\left(x_{i}\right) \cdot i_{R}^{n}\left(x_{i}\right)+d_{P}^{n}\left(x_{i}\right) \cdot d_{R}^{n}\left(x_{i}\right)}{\sqrt{\left(s_{P}^{n}\left(x_{i}\right)\right)^{2}+\left(i_{P}^{n}\left(x_{i}\right)\right)^{2}+\left(d_{P}^{n}\left(x_{i}\right)\right)^{2}} \cdot \sqrt{\left(s_{R}^{n}\left(x_{i}\right)\right)^{2}+\left(i_{R}^{n}\left(x_{i}\right)\right)^{2}+\left(d_{R}^{n}\left(x_{i}\right)\right)^{2}}}
\end{aligned}
$$

If $P=\left[s_{P}\left(x_{i}\right), i_{P}\left(x_{i}\right), d_{P}\left(x_{i}\right)\right], Q=\left[s_{Q}\left(x_{i}\right), i_{Q}\left(x_{i}\right), d_{Q}\left(x_{i}\right)\right]$ and $R=\left[s_{Q}\left(x_{i}\right), i_{Q}\left(x_{i}\right), d_{Q}\left(x_{i}\right)\right]$ are considered as three vectors in a plane such that $P\left(x_{i}\right) \sqsubseteq Q\left(x_{i}\right) \sqsubseteq R\left(x_{i}\right)$ and using triangular inequality, we have $\mathrm{d}^{\prime}\left(P\left(x_{i}\right), R\left(x_{i}\right)\right) \leq \mathrm{d}^{\prime}\left(P\left(x_{i}\right), Q\left(x_{i}\right)\right)+\mathrm{d}^{\prime}\left(Q\left(x_{i}\right), R\left(x_{i}\right)\right)$ and hence (4) holds true. 
In the following, $w=\left(w_{1}, w_{2}, w_{3} \ldots w_{m}\right)^{T}$ represents a weight vector such that $w_{i} \in[0,1]$ and $\sum_{i=1}^{m} w_{i}=1$

Definition 14. For two TSFNs $P=\left(s_{P}, i_{P}, d_{P}\right)$ and $Q=\left(s_{Q}, i_{Q}, d_{Q}\right)$ on a universe of discourse $X, a$ weighted cosine similarity measure is defined as:

$$
\omega C_{T S F S}^{1}(P, Q)=\frac{1}{m} \sum_{i=1}^{m} w_{i} \frac{s_{P}^{n}\left(x_{i}\right) \cdot s_{Q}^{n}\left(x_{i}\right)+i_{P}^{n}\left(x_{i}\right) \cdot i_{Q}^{n}\left(x_{i}\right)+d_{P}^{n}\left(x_{i}\right) \cdot d_{Q}^{n}\left(x_{i}\right)}{\sqrt{\left(s_{P}^{n}\left(x_{i}\right)\right)^{2}+\left(i_{P}^{n}\left(x_{i}\right)\right)^{2}+\left(d_{P}^{n}\left(x_{i}\right)\right)^{2}} \cdot \sqrt{\left(s_{Q}^{n}\left(x_{i}\right)\right)^{2}+\left(i_{Q}^{n}\left(x_{i}\right)\right)^{2}+\left(d_{Q}^{n}\left(x_{i}\right)\right)^{2}}}
$$

By taking $w_{i}=\frac{1}{m}$ the Equation (8) reduces to Equation (7).

The following properties hold true for the weighted cosine similarity measures of three TSFNs $P=$ $\left(s_{P}, i_{P}, d_{P}\right), Q=\left(s_{Q}, i_{Q}, d_{Q}\right)$ and $R=\left(s_{R}, i_{R}, d_{R}\right)$.

1. $0 \leq W_{\text {TSFS }}^{1}(P, Q) \leq 1$.

2. $W_{C ̧ C S F S}^{1}(P, Q)=W C_{T S F S}^{1}(Q, P)$.

3. $\quad W C_{\text {TSFS }}^{1}(P, Q)=1$ iff $P=Q$ and $i=1,2,3, \ldots m$.

4. If $P \sqsubseteq Q \sqsubseteq R$. Then $\mathrm{WÇ}_{T S F S}^{1}(P, R) \leq \mathrm{WÇ}_{T S F S}^{1}(P, Q)$, $\operatorname{WÇSFS}_{T S}^{1}(P, R) \leq W C_{T S F S}^{1}(Q, R)$.

Proof. Proofs are straightforward.

\subsection{Set-Theoretic Similarity Measures}

For two TSFNs, in view of $[28,37,40]$ some set-theoretic similarity measures and weighted set-theoretic similarity measures are developed in this section.

Definition 15. For two TSFNs $P=\left(s_{P}, i_{P}, d_{P}\right)$ and $Q=\left(s_{Q}, i_{Q}, d_{Q}\right)$ on a universe of discourse $X, a$ set-theoretic similarity measure is defined as:

$$
C_{\zeta T S F S}^{2}(P, Q)=\frac{1}{m} \sum_{i=1}^{m} \frac{s_{P}^{n}\left(x_{i}\right) \cdot s_{Q}^{n}\left(x_{i}\right)+i_{P}^{n}\left(x_{i}\right) \cdot i_{Q}^{n}\left(x_{i}\right)+d_{P}^{n}\left(x_{i}\right) \cdot d_{Q}^{n}\left(x_{i}\right)}{\max \left(\left(s_{P}^{n}\left(x_{i}\right)\right)^{2}+\left(i_{P}^{n}\left(x_{i}\right)\right)^{2}+\left(d_{P}^{n}\left(x_{i}\right)\right)^{2},\left(s_{Q}^{n}\left(x_{i}\right)\right)^{2}+\left(i_{Q}^{n}\left(x_{i}\right)\right)^{2}+\left(d_{Q}^{n}\left(x_{i}\right)\right)^{2}\right)}
$$

Definition 16. For two TSFNs $P=\left(s_{P}, i_{P}, d_{P}\right)$ and $Q=\left(s_{Q}, i_{Q}, d_{Q}\right)$ on a universe of discourse $X, a$ weighted set-theoretic similarity measure is defined as:

$$
\omega C_{T S F S}^{2}(P, Q)=\frac{1}{m} \sum_{i=1}^{m} w_{i} \frac{s_{P}^{n}\left(x_{i}\right) \cdot s_{Q}^{n}\left(x_{i}\right)+i_{P}^{n}\left(x_{i}\right) \cdot i_{Q}^{n}\left(x_{i}\right)+d_{P}^{n}\left(x_{i}\right) \cdot d_{Q}^{n}\left(x_{i}\right)}{\max \left(\left(s_{P}^{n}\left(x_{i}\right)\right)^{2}+\left(i_{P}^{n}\left(x_{i}\right)\right)^{2}+\left(d_{P}^{n}\left(x_{i}\right)\right)^{2},\left(s_{Q}^{n}\left(x_{i}\right)\right)^{2}+\left(i_{Q}^{n}\left(x_{i}\right)\right)^{2}+\left(d_{Q}^{n}\left(x_{i}\right)\right)^{2}\right)}
$$

Equation (10) reduces to Equation (9) if we place $w_{i}=\frac{1}{m}$.

The following properties hold true for the set-theoretic similarity measure of two TSFNs $P=\left(s_{P}, i_{P}, d_{P}\right)$, $Q=\left(s_{Q}, i_{Q}, d_{Q}\right)$.

1. $0 \leq C_{T S F S}^{2}(P, Q) \leq 1$.

2. $\quad C_{T S F S}^{2}(P, Q)=C_{T S F S}^{2}(Q, P)$.

3. $C_{T S F S}^{2}(P, Q)=1$ if $P=Q$ and $i=1,2,3, \ldots m$.

The following three properties hold true for the weighted set-theoretic similarity measure of two TSFNs $P=\left(s_{P}, i_{P}, d_{P}\right), Q=\left(s_{Q}, i_{Q}, d_{Q}\right)$ and $R=\left(s_{R}, i_{R}, d_{R}\right)$. 
1. $0 \leq W_{T S F S}^{2}(P, Q) \leq 1$.

2. $\quad W C_{T S F S}^{2}(P, Q)=W C_{T S F S}^{2}(Q, P)$.

3. $W_{T S F S}^{2}(P, Q)=1$ if $P=Q$ and $i=1,2,3, \ldots m$.

\subsection{Grey Similarity Measure}

Following, the similarity measures proposed in References $[28,37,40]$ and the grey similarity measures in the environment of TSFSs are developed as a generalization of existing grey similarity measures defined in Equations (3) and (6).

Definition 17. For two TSFNs $P=\left(s_{P}, i_{P}, d_{P}\right)$ and $Q=\left(s_{Q}, i_{Q}, d_{Q}\right)$ on a universe of discourse $X$, the grey similarity measure is defined as:

$$
C_{T S F S}^{3}(P, Q)=\frac{1}{3 m} \sum_{i=1}^{m}\left(\frac{\Delta s_{\min }+\Delta s_{\max }}{\Delta s_{i}+\Delta s_{\max }}+\frac{\Delta i_{\min }+\Delta i_{\max }}{\Delta i_{i}+\Delta i_{\max }}+\frac{\Delta d_{\min }+\Delta d_{\max }}{\Delta d_{i}+\Delta d_{\max }}\right)
$$

where $\Delta s_{i}^{n}=\left|s_{P}^{n}\left(x_{i}\right)-s_{Q}^{n}\left(x_{i}\right)\right|, \Delta i_{i}^{n}=\left|i_{P}^{n}\left(x_{i}\right)-i_{Q}^{n}\left(x_{i}\right)\right|$ and $\Delta d_{i}^{n}=\left|d_{P}^{n}\left(x_{i}\right)-d_{Q}^{n}\left(x_{i}\right)\right|$ further $\Delta s_{\text {min }}^{n}=$ $\min \left\{\left|s_{P}^{n}\left(x_{i}\right)-s_{Q}^{n}\left(x_{i}\right)\right|\right\}, \Delta i_{\text {min }}^{n}=\min \left\{\left|i_{P}^{n}\left(x_{i}\right)-i_{Q}^{n}\left(x_{i}\right)\right|\right\}$ and $\Delta d_{\text {min }}^{n}=\min \left\{\left|d_{P}^{n}\left(x_{i}\right)-d_{Q}^{n}\left(x_{i}\right)\right|\right\}$ also $\Delta s_{\max }^{n}=\max \left\{\left|s_{P}^{n}\left(x_{i}\right)-s_{Q}^{n}\left(x_{i}\right)\right|\right\}, \Delta i_{\max }^{n}=\max \left\{\left|i_{P}^{n}\left(x_{i}\right)-i_{Q}^{n}\left(x_{i}\right)\right|\right\}$ and $\Delta d_{\max }^{n}=$ $\max \left\{\left|d_{P}^{n}\left(x_{i}\right)-d_{Q}^{n}\left(x_{i}\right)\right|\right\}$.

The following properties hold true for the Grey similarity measure of two TSFNS $P=\left(s_{P}, i_{P}, d_{P}\right)$, $Q=\left(s_{Q}, i_{Q}, d_{Q}\right)$.

1. $0 \leq \mathrm{C}_{T S F S}^{3}(P, Q) \leq 1$.

2. $C_{T S F S}^{3}(P, Q)=C_{T S F S}^{3}(Q, P)$.

3. $C_{3 T S S}^{3}(P, Q)=1$ if $P=Q$ and $i=1,2,3, \ldots m$.

Whenever the weight of the elements is considered as a real-life phenomenon, attributes have different importance in different situations and therefore need to be weighted. Consequently, we developed weighted similarity measures described as follows:

Definition 18. For two TSFNs $P=\left(s_{P}, i_{P}, d_{P}\right)$ and $Q=\left(s_{Q}, i_{Q}, d_{Q}\right)$ on a universe of discourse $X$, the weighted grey similarity measure is defined as:

$$
\omega C_{\text {TSFS }}^{3}(P, Q)=\frac{1}{3} \sum_{i=1}^{m} w_{i}\left(\frac{\Delta s_{\text {min }}+\Delta s_{\text {max }}}{\Delta s_{i}+\Delta s_{\max }}+\frac{\Delta i_{\text {min }}+\Delta i_{\text {max }}}{\Delta i_{i}+\Delta i_{\text {max }}}+\frac{\Delta d_{\text {min }}+\Delta d_{\text {max }}}{\Delta d_{i}+\Delta d_{\text {max }}}\right)
$$

where $\Delta s_{i}^{n}=\left|s_{P}^{n}\left(x_{i}\right)-s_{Q}^{n}\left(x_{i}\right)\right|, \Delta i_{i}^{n}=\left|i_{P}^{n}\left(x_{i}\right)-i_{Q}^{n}\left(x_{i}\right)\right|$ and $\Delta d_{i}^{n}=\left|d_{P}^{n}\left(x_{i}\right)-d_{Q}^{n}\left(x_{i}\right)\right|$ further $\Delta s_{\text {min }}^{n}=$ $\min \left\{\left|s_{P}^{n}\left(x_{i}\right)-s_{Q}^{n}\left(x_{i}\right)\right|\right\}, \Delta i_{\text {min }}^{n}=\min \left\{\left|i_{P}^{n}\left(x_{i}\right)-i_{Q}^{n}\left(x_{i}\right)\right|\right\}$ and $\Delta d_{\min }^{n}=\min \left\{\left|d_{P}^{n}\left(x_{i}\right)-d_{Q}^{n}\left(x_{i}\right)\right|\right\}$ also $\Delta s_{\max }^{n}=\max \left\{\left|s_{P}^{n}\left(x_{i}\right)-s_{Q}^{n}\left(x_{i}\right)\right|\right\}, \Delta i_{\max }^{n}=\max \left\{\left|i_{P}^{n}\left(x_{i}\right)-i_{Q}^{n}\left(x_{i}\right)\right|\right\}$ and $\Delta d_{\max }^{n}=$ $\max \left\{\left|d_{P}^{n}\left(x_{i}\right)-d_{Q}^{n}\left(x_{i}\right)\right|\right\}$.

If we consider $w_{i}=\frac{1}{m}$, Equations (12) reduces to Equation (11).

The following properties hold true for the weighted grey similarity measure of two TSFNs $P=\left(s_{P}, i_{P}, d_{P}\right)$, $Q=\left(s_{Q}, i_{Q}, d_{Q}\right)$.

1. $0 \leq \operatorname{WÇ}_{T S F S}^{3}(P, Q) \leq 1$.

2. $\quad W C_{\text {TSFS }}^{3}(P, Q)=W C_{T S F S}^{3}(Q, P)$.

3. $\quad \operatorname{WC}_{T S F S}^{3}(P, Q)=1$ if $P=Q$ and $i=1,2,3, \ldots m$. 
In this section, we have noted that the proposed similarity measures are a generalization of all existing relevant similarity measures, i.e., the proposed similarity measures can deal with any type of data that could be handled with similarity measures of IFSs and PFSs, but conversely, the similarity measures of IFSs and PFSs are unable to handle data in the environment of TSFSs.

\section{Application in Building Material Recognition}

The tools of similarity measures have applications in pattern classification. In such a phenomenon, the class of an unknown pattern or object is found using some similarity measuring tools and some preferences of decision makers. In this section, the similarity measures developed so far in Section 3 are applied to a building material recognition problem where the class of an unknown building material needed to be evaluated. The results obtained using the similarity measures of TSFSs are then analyzed for description of the advantages of proposed work and the limitations of existing work. To explain the phenomenon, an illustrative example adapted from Reference [40] is discussed.

Example 1. Consider TSFNs $P_{i}(i=1,2,3,4)$ representing four building materials and let $X=$ $\left\{x_{i}: i=1,2,3, \ldots 7\right\}$ be the space of attributes having weights $w=(0.16,0.12,0.09,0.18,0.20,0.10,0.15)^{T}$. We assume another unknown material $P$ with the hypothetical information listed in Table 1 . With the help of defined similarity measures for TSFSs we shall identify the class of unknown building material from four materials denoted by $P_{i}(i=1,2,3,4)$. Using the recognition principle developed in $[28,40]$, the evaluation of class of $P$ to $P_{i}$ is established.

$$
k=\arg \operatorname{Max}_{1 \leq i \leq 4}\left\{O C_{T S F S}\right\}
$$

All the numbers in Table 1 are purely TSFNs for $n=4$ which means that the tools of IFSs and PFSs could not handle such type of data as their structures are limited. Even SFSs could not handle this type of data as in SFSs we have $n=2$. This shows the strength and diverse nature of TSFSs. Now, the different similarity measuring tools defined in Section 3 are applied to the given data in Table 1 and the results are provided in Table 2.

Table 1. Data on building materials.

\begin{tabular}{cccccc}
\hline & $\boldsymbol{P}_{\mathbf{1}}$ & $\boldsymbol{P}_{\mathbf{2}}$ & $\boldsymbol{P}_{\mathbf{3}}$ & $\boldsymbol{P}_{\mathbf{4}}$ & $\boldsymbol{P}$ \\
\hline $\boldsymbol{x}_{\mathbf{1}}$ & $(0.56,0.47,0.22)$ & $(0.81,0.3,0.37)$ & $(0.43,0.43,0.55)$ & $(0.57,0.51,0.39)$ & $(0.34,0.56,0.78)$ \\
$\boldsymbol{x}_{\mathbf{2}}$ & $(0.11,0.11,0.11)$ & $(0.59,0.66,0.66)$ & $(0.91,0.34,0.68)$ & $(0.56,0.76,0.31)$ & $(0.47,0.38,0.84)$ \\
$\boldsymbol{x}_{\mathbf{3}}$ & $(0.35,0.45,0.61)$ & $(0.42,0.56,0.71)$ & $(0.81,0.41,0.35)$ & $(0.27,0.59,0.72)$ & $(0.55,0.44,0.65)$ \\
$x_{\mathbf{4}}$ & $(0.33,0.54,0.31)$ & $(0.59,0.45,0.9)$ & $(0.44,0.55,0.77)$ & $(0.46,0.46,0.45)$ & $(0.76,0.46,0.85)$ \\
$x_{5}$ & $(0.35,0.2,0.64)$ & $(0.16,0.33,0.42)$ & $(0.55,0.44,0.29)$ & $(0.57,0.66,0.91)$ & $(0.13,0.35,0.57)$ \\
$x_{\mathbf{6}}$ & $(0.47,0.37,0.68)$ & $(0.68,0.46,0.88)$ & $(0.47,0.66,0.75)$ & $(0.41,0.73,0.41)$ & $(0.24,0.54,0.45)$ \\
$x_{7}$ & $(0.78,0.55,0.03)$ & $(0.49,0.54,0.39)$ & $(0.58,0.34,0.23)$ & $(0.21,0.43,0.13)$ & $(0.82,0.46,0.69)$ \\
\hline
\end{tabular}

Table 2. Similarity Measures of $P_{i}$ with $P$.

\begin{tabular}{ccccc}
\hline Similarity Measures & $\left(\boldsymbol{P}_{\mathbf{1}}, \boldsymbol{P}\right)$ & $\left(\boldsymbol{P}_{\mathbf{2}}, \boldsymbol{P}\right)$ & $\left(\boldsymbol{P}_{\mathbf{3}}, \boldsymbol{P}\right)$ & $\left(\boldsymbol{P}_{\mathbf{4}}, \boldsymbol{P}\right)$ \\
\hline UÇ TSSF & 0.612207 & 0.690072 & 0.64601 & 0.603693 \\
WÇTSSS & 0.3197 & 0.367149 & 0.26296 & 0.160122 \\
WÇTSFS & 0.762518 & 0.792809 & 0.796319 & 0.750893 \\
\hline
\end{tabular}

Analyzing Table 2, it seems that material $P_{2}$ is close to $P$ as the similarity measure of $\left(P_{2}, P\right)$ is larger than of all other pairs if we apply cosine or set theoretic similarity measure. However, if we apply grey similarity measure, it seems that the values of $\left(P_{2}, P\right)$ and $\left(P_{3}, P\right)$ can be considered nearly equal or the similarity of $\left(P_{3}, P\right)$ is slightly higher than that of $\left(P_{2}, P\right)$, so by grey similarity measure, $P$ has a relatively larger similarity measure to the class of $P_{3}$. Thus, it is concluded that based on cosine or set-theoretic similarity measure, the unknown material $P$ belongs to the class of $P_{2}$ type material while based on grey similarity measure, the material $P$ belongs to $P_{3}$ type material. 


\section{Comparative Study and Advantages}

The similarity measures developed in this manuscript are generalizations of similarity measures proposed in $[28,37,40]$. The following remarks proved that the similarity measures defined in Equations (7)-(12) are generalizations of the similarity measures of References $[28,37,40]$.

Remark 2. By placing $n=2$, Equations (7) and (8) reduce to similarity measures of SFSs and by placing $n=1$, Equations (7) and (8) reduce to similarity measures of PFSs [40]. Similarly, placement of $i_{P}=i_{Q}=0$ and $n=1$ reduces Equations (7) and (8) to similarity measures for IFSs [28]. This shows that similarity measures proposed in Equations (7) and (8) are generalizations of existing similarity measures.

Remark 3. By placing $n=2$, Equations (9) and (10) reduce to similarity measures of SFSs and by placing $n=1$, Equations (9) and (10) reduce to similarity measures of PFSs [40]. Similarly, placement of $r_{P}=r_{Q}=0$ and $n=1$ reduces Equations (9) and (10) to similarity measures of IFSs [37]. This shows that similarity measures proposed in Equations (9) and (10) are generalizations of existing similarity measures.

Remark 4. By placing $n=2$, Equations (11) and (12) reduce to similarity measures of SFSs and by placing $n=1$, Equations (11) and (12) reduce to similarity measures of PFSs [40]. Similarly, placement of $r_{P}=r_{Q}=0$ and $n=1$ reduces Equations (11) and (12) to similarity measures of IFSs [37]. This shows that similarity measures proposed in Equations (11) and (12) are generalizations of existing similarity measures.

The main advantage of the similarity measures proposed in the environment of SFSs and TSFSs is that these similarity measures can handle the data provided in References [28,37,40].

Now, the building material recognition problem from Reference [40] is solved using similarity measures for TSFSs for $n=1$.

Example 2. In this problem from [40], four building materials are denoted by $P_{i}(i=1,2,3,4)$. The weighted similarity measures of TSFSs defined in Equations (8), (10) and (12) are applied on the data provided in Table 3 to evaluate the class of unknown building material $P$. The weight vector in this case is $(0.12,0.15,0.09,0.16,0.20,0.10,0.18)^{T}$.

All data in Table 3 is picture fuzzy environment, so using the similarity measures defined in Equations (8), (10), and (12) and taking $n=1$ results in:

Table 3. Data of Patterns [40].

\begin{tabular}{cccccc}
\hline & $\boldsymbol{P}_{\mathbf{1}}$ & $\boldsymbol{P}_{\mathbf{2}}$ & $\boldsymbol{P}_{\mathbf{3}}$ & $\boldsymbol{P}_{\mathbf{4}}$ & $\boldsymbol{P}$ \\
\hline $\boldsymbol{x}_{\mathbf{1}}$ & $(0.17,0.53,0.13)$ & $(0.51,0.24,0.21)$ & $(0.31,0.39,0.25)$ & $(1,0,0)$ & $(0.91,0.03,0.05)$ \\
$\boldsymbol{x}_{\mathbf{2}}$ & $(0.10,0.81,0.05)$ & $(0.62,0.12,0.07)$ & $(0.60,0.26,0.11)$ & $(1,0,0)$ & $(0.78,0.12,0.07)$ \\
$x_{\mathbf{3}}$ & $(0.53,0.33,0.09)$ & $(1,0,0)$ & $(0.91,0.03,0.02)$ & $(0.85,0.09,0.05)$ & $(0.90,0.05,0.02)$ \\
$\boldsymbol{x}_{\mathbf{4}}$ & $(0.89,0.08,0.03)$ & $(0.13,0.64,0.21)$ & $(0.07,0.09,0.07)$ & $(0.74,0.16,0.1)$ & $(0.68,0.08,0.21)$ \\
$x_{5}$ & $(0.42,0.35,0.18)$ & $(0.03,0.82,0.13)$ & $(0.04,0.85,0.10)$ & $(0.02,0.89,0.05)$ & $(0.05,0.87,0.06)$ \\
$x_{\mathbf{6}}$ & $(0.08,0.89,0.02)$ & $(0.73,0.15,0.08)$ & $(0.68,0.26,0.06)$ & $(0.08,0.84,0.06)$ & $(0.13,0.75,0.09)$ \\
$x_{\mathbf{7}}$ & $(0.33,0.51,0.12)$ & $(0.52,0.31,0.16)$ & $(0.15,0.76,0.07)$ & $(0.16,0.71,0.05)$ & $(0.15,0.73,0.08)$ \\
\hline
\end{tabular}

The results obtained in Table 4 are similar to those obtained in Reference [40] which strengthened our claim that the similarity measures of TSFSs can handle the data provided in the environment of PFSs. Similarly, by placing $i_{A}=i_{B}=0$ and $n=1$ it is claimed that the similarity measures defined in Equations (7)-(12) could handle the data provided in the environment of IFSs. Conversely, the similarity measures of IFSs and PFSs could not handle the data provided in TSF environment as a TSFN cannot be considered as an IFN or PFN in general. 
Table 4. Similarity Measures of $P_{i}$ with $P$.

\begin{tabular}{ccccc}
\hline Similarity Measures & $\left(\boldsymbol{P}_{\mathbf{1}}, \boldsymbol{P}\right)$ & $\left(\boldsymbol{P}_{\mathbf{2}}, \boldsymbol{P}\right)$ & $\left(\boldsymbol{P}_{\mathbf{3}}, \boldsymbol{P}\right)$ & $\left(\boldsymbol{P}_{\mathbf{4}}, \boldsymbol{P}\right)$ \\
\hline UÇ TSSS $_{\text {TCF }}^{1}$ & 0.715235 & 0.763072 & 0.855508 & 0.993654 \\
WÇSS & 0.5556 & 0.65557 & 0.693305 & 0.919909 \\
WÇTSFS & 0.708292 & 0.785837 & 0.915441 & 0.941545 \\
\hline
\end{tabular}

Another advantage of proposed similarity measures is that in the environment of TSFSs there is no restriction for assigning values to membership functions while in the environment of IFSs, Pythagorean FSs and PFSs some restrictions in assigning membership values as described in Section 1 must be faced.

\section{Conclusions}

This article described the background of IFSs, Pythagorean FSs and PFSs in detail for observing the limited nature of their structures. It is discussed how the shortcomings that exists in current structures are improved using the framework of SFSs and TSFSs with the help of diagrams and numerical examples. Then, some similarity measures of IFSs and PFSs have been observed and their limitations were discussed. To generalize these similarity measures, some new similarity measures were developed. The generalization of new similarity measures was discussed with the help of some remarks and examples showing its diverse structure. The new similarity measures of TSFSs was applied to a problem of building material recognition where the information was provided in form of PFNs and the results obtained was found to be similar to previously existing results. Another problem in the environment of TSFSs was solved using new similarity measures and it was discussed that the existing similarity measures could not handle this type of data. The idea of SFSs and TSFSs is novel and some other tools of similarity and distance measures, correlation coefficients could be developed in near future. Further, some aggregation operators could be developed for TSFSs and applied in decision making problems, clustering problems, etc.

Author Contributions: Conceptualization, K.U., T.M. and N.J.; Data curation, K.U., T.M. and N.J.; Investigation, K.U., T.M. and N.J.; Methodology K.U., T.M. and N.J.; Software, K.U., T.M. and N.J.; Validation, K.U., T.M. and N.J.; Writing—original draft, K.U., T.M. and N.J.; Writing—review \& editing, K.U., T.M. and N.J.

Acknowledgments: The authors are highly thankful to the editor and the anonymous reviewers for their valuable suggestions and comments. This work was supported by Higher Education Commission, Pakistan under National Research Program for Universities (NRPU), Project No. 5833.

Conflicts of Interest: The authors declare no conflict of interest.

\section{References}

1. Zadeh, L.A. Information and control. Fuzzy Sets 1965, 8, 338-353.

2. Atanassov, K.T. Intuitionistic fuzzy sets. Fuzzy Sets Syst. 1986, 20, 87-96. [CrossRef]

3. Yager, R.R. Pythagorean fuzzy subsets. In Proceedings of the 2013 Joint IFSA World Congress and NAFIPS Annual Meeting (IFSA/NAFIPS), Edmonton, AB, Canada, 24-28 June 2013.

4. Yager, R.R.; Abbasov, A.M. Pythagorean membership grades, complex numbers, and decision making. Int. J. Intell. Syst. 2013, 28, 436-452. [CrossRef]

5. Atanassov, K.T. Intuitionistic fuzzy sets. In Intuitionistic Fuzzy Sets; Springer: Berlin/Heidelberg, Germany, 1999; pp. 1-137.

6. Atanassov, K.T. More on intuitionistic fuzzy sets. Fuzzy Sets Syst. 1989, 33, 37-45. [CrossRef]

7. Szmidt, E.; Kacprzyk, J. Distances between intuitionistic fuzzy sets. Fuzzy Sets Syst. 2000, 114, 505-518. [CrossRef]

8. $\mathrm{Xu}, \mathrm{Z}$;; Yager, R.R. Some geometric aggregation operators based on intuitionistic fuzzy sets. Int. J. Gen. Syst. 2006, 35, 417-433. [CrossRef]

9. Peng, X.; Yang, Y. Some results for Pythagorean fuzzy sets. Int. J. Intell. Syst. 2015, 30, 1133-1160. [CrossRef] 
10. Garg, H. A novel accuracy function under interval-valued Pythagorean fuzzy environment for solving multicriteria decision making problem. J. Intell. Fuzzy Syst. 2016, 31, 529-540. [CrossRef]

11. Cuong, B. Picture Fuzzy Sets-First Results. Part 1. Preprint 03/2013 and Preprint 04/2013; Institute of Mathematics: Hanoi, Vietnam, 2013.

12. Cường, B.C. Picture fuzzy sets. J. Comput. Sci. Cybern. 2014, 30, 409-420. [CrossRef]

13. Wei, G. Picture fuzzy cross-entropy for multiple attribute decision making problems. J. Bus. Econ. Manag. 2016, 17, 491-502. [CrossRef]

14. Singh, P. Correlation coefficients for picture fuzzy sets. J. Intell. Fuzzy Syst. 2015, 28, 591-604.

15. Thong, P.H. A new approach to multi-variable fuzzy forecasting using picture fuzzy clustering and picture fuzzy rule interpolation method. In Knowledge and Systems Engineering; Springer: Berlin/Heidelberg, Germany, 2015; pp. 679-690.

16. Cuong, B.C.; Van Hai, P. Some fuzzy logic operators for picture fuzzy sets. In Proceedings of the 2015 Seventh International Conference on Knowledge and Systems Engineering (KSE), Ho Chi Minh City, Vietnam, 8-10 October 2015.

17. Cuong, B.C.; Kreinovitch, V.; Ngan, R.T. A classification of representable t-norm operators for picture fuzzy sets. In Proceedings of the 2016 Eighth International Conference on Knowledge and Systems Engineering (KSE), Hanoi, Vietnam, 6-8 October 2016.

18. Mahmood, T.; Ullah, K.; Khan, Q.; Jan, N. An approach toward decision-making and medical diagnosis problems using the concept of spherical fuzzy sets. Neural Comput Appl. 2018, 1-13. [CrossRef]

19. Yang, M.-S.; Lin, D.-C. On similarity and inclusion measures between type-2 fuzzy sets with an application to clustering. Comput. Math. Appl. 2009, 57, 896-907. [CrossRef]

20. De Oliveira, J.V.; Pedrycz, W. Advances in Fuzzy Clustering and Its Applications; John Wiley \& Sons: Hoboken, NJ, USA, 2007.

21. Boriah, S.; Chandola, V.; Kumar, V. Similarity measures for categorical data: A comparative evaluation. In Proceedings of the 2008 SIAM International Conference on Data Mining, Atlanta, GA, USA, 24-26 April 2008.

22. Nasraoui, O.; Frigui, H.; Joshi, A.; Krishnapuram, R. Mining web access logs using relational competitive fuzzy clustering. In Proceedings of the Eight International Fuzzy Systems Association World Congress, Taipei, Taiwan, 17-20 August 1999.

23. Dengfeng, L.; Chuntian, C. New similarity measures of intuitionistic fuzzy sets and application to pattern recognitions. Pattern Recognit. Lett. 2002, 23, 221-225. [CrossRef]

24. Hung, W.-L.; Yang, M.-S. Similarity measures of intuitionistic fuzzy sets based on Hausdorff distance. Pattern Recognit. Lett. 2004, 25, 1603-1611. [CrossRef]

25. Kandel, A. Fuzzy Techniques in Pattern Recognition; John Wiley \& Sons: Hoboken, NJ, USA, 1982.

26. Liang, Z.; Shi, P. Similarity measures on intuitionistic fuzzy sets. Pattern Recognit. Lett. 2003, 24, $2687-2693$. [CrossRef]

27. Vlachos, I.K.; Sergiadis, G.D. Intuitionistic fuzzy information-applications to pattern recognition. Pattern Recognit. Lett. 2007, 28, 197-206. [CrossRef]

28. Ye, J. Cosine similarity measures for intuitionistic fuzzy sets and their applications. Math. Comput. Model. 2011, 53, 91-97. [CrossRef]

29. Mitchell, H.B. Pattern recognition using type-II fuzzy sets. Inf. Sci. 2005, 170, 409-418. [CrossRef]

30. Han, J.; Ma, K.-K. Fuzzy color histogram and its use in color image retrieval. IEEE Trans. Image Process. 2002, 11, 944-952. [CrossRef] [PubMed]

31. Liao, T.W.; Zhang, Z.; Mount, C.R. Similarity measures for retrieval in case-based reasoning systems. Appl. Artif. Intell. 1998, 12, 267-288. [CrossRef]

32. Santini, S.; Jain, R. Similarity measures. IEEE Trans. Pattern Anal. Mach. Intell. 1999, 21, 871-883. [CrossRef]

33. Szmidt, E.; Kacprzyk, J. A similarity measure for intuitionistic fuzzy sets and its application in supporting medical diagnostic reasoning. In Proceedings of the International Conference on Artificial Intelligence and Soft Computing, Zakopane, Poland, 7-11 June 2004; Springer: Berlin/Heidelberg, Germany, 2004.

34. $\mathrm{Xu}, \mathrm{Z}$. Some similarity measures of intuitionistic fuzzy sets and their applications to multiple attribute decision making. Fuzzy Optim. Decis. Mak. 2007, 6, 109-121. [CrossRef]

35. $\mathrm{Xu}, \mathrm{Z}$; Chen, J. An overview of distance and similarity measures of intuitionistic fuzzy sets. Int. J. Uncertain. Fuzz. Knowl.-Based Syst. 2008, 16, 529-555. [CrossRef] 
36. $\mathrm{Xu}, \mathrm{Z}$. On correlation measures of intuitionistic fuzzy sets. In Proceedings of the International Conference on Intelligent Data Engineering and Automated Learning, Burgos, Spain, 20-23 September 2006; Springer: Berlin/Heidelberg, Germany, 2006.

37. Xu, Z.; Cai, X. Correlation, Distance and Similarity Measures of Intuitionistic Fuzzy Sets. In Intuitionistic Fuzzy Information Aggregation; Springer: Berlin, Heidelberg, 2012; pp. 151-188.

38. Wei, G.; Lin, R.; Wang, H. Distance and similarity measures for hesitant interval-valued fuzzy sets. J. Intell. Fuzzy Syst. 2014, 27, 19-36.

39. Mitchell, H. A correlation coefficient for intuitionistic fuzzy sets. Int. J. Intell. Syst. 2004, 19, 483-490. [CrossRef]

40. Wei, G. Some similarity measures for picture fuzzy sets and their applications. Iran. J. Fuzzy Syst. 2018, 15, 77-89.

41. Son, L.H. Generalized picture distance measure and applications to picture fuzzy clustering. Appl. Soft Comput. 2016, 46, 284-295. [CrossRef]

(C) 2018 by the authors. Licensee MDPI, Basel, Switzerland. This article is an open access article distributed under the terms and conditions of the Creative Commons Attribution (CC BY) license (http://creativecommons.org/licenses/by/4.0/). 